\title{
Eficiencia en el consumo de diésel de un camión con la implementación de un dispositivo aerodinámico
}

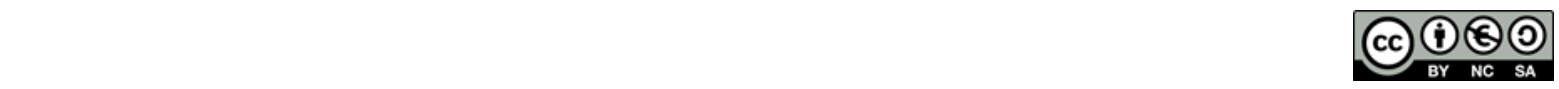

Efficiency in diesel consumption of a truck with the implementation of an aerodynamic device

David Paul Pachacama Gualotuña. ${ }^{1}$, Darwin Anderson Pachacama Gualotuña. ${ }^{2}$, Lenin Paul Vinlasaca Viera. ${ }^{3}, \&$ Antonio Gabriel Castillo Medina. ${ }^{4}$

Recibido: 10-02-2020 / Revisado: 22-02-2020 /Aceptado: 04-03-2020/ Publicado: 03-04-2020

\begin{abstract}
.
https://doi.org/10.33262/cienciadigital.v4i2.1193

The research evaluates the impact that an aerodynamic device has on the diesel consumption of a medium-sized truck, by studying the drag force generated by the vehicle when it moves forward, the aerodynamic coefficient (drag) is considered, a Of the important variables that influence the reduction of this force, this coefficient has a dimensionless value of 0.78 in trucks according to international standards, with the use of several aerodynamic devices that can be incorporated in the different parts of the truck, so that the flow of air both outside and inside generate the least amount of restriction when traveling on the surfaces of the truck, this reduction would be achieved above, to assess the efficiency in fuel consumption, use DIN 70 030-2 through which a test protocol is developed, among the requirements of the standard is that the truck must travel $10[\mathrm{~km}]$ at a constant speed of $90[\mathrm{~km} / \mathrm{h}]$, with a minimum of 4 repetitions and a maximum of 10 , the diesel consumption was measured by gravimetry and with the Hino diagnostic equipment. The result of the investigation is a saving of 4.63 [\%] in diesel consumption.
\end{abstract}

Keywords: Efficiency, Drag force, Drag coefficient, Fuel consumption, Gravimetry.

\footnotetext{
${ }^{1}$ Universidad de las Fuerzas Armadas ESPE, Pichincha, Ecuador, dppachacama1 @espe.edu.ec

${ }^{2}$ Universidad de las Fuerzas Armadas ESPE, Pichincha, Ecuador, dapachacama@espe.edu.ec

${ }^{3}$ Universidad de las Fuerzas Armadas ESPE, Pichincha, Ecuador, lpvinlasaca@espe.edu.ec

${ }^{4}$ Escuela Superior Politécnica del Chimborazo, Riobamba, Ecuador, antoniog.castillo@espoch.edu.ec
} 


\section{Resumen.}

La investigación evalúa el impacto que tiene un dispositivo aerodinámico en el consumo de diesel de un camión de carga mediana, mediante el estudio de la fuerza de arrastre que genera el vehículo cuando se desplaza hacia adelante, se considera al coeficiente aerodinámico (arrastre), una de las variables importantes que influye en la reducción de esta fuerza, este coeficiente tiene un valor adimensional de 0,78 en camiones según normas internaciones, con el uso de varios dispositivos aerodinámicos que se pueden incorporar en las distintas partes del camión, de manera que el flujo de aire tanto exterior como interior genere la menor cantidad de restricción al momento de circular por las superficies del camión, se lograría esta reducción antes mencionada, para evaluar la eficiencia en el consumo de combustible se recurre a la norma DIN 70 030-2 mediante la cual se elabora un protocolo de prueba, entre los requerimientos de la norma se encuentra que el camión debe recorrer $10[\mathrm{~km}]$ a una velocidad constante de $90[\mathrm{~km} / \mathrm{h}]$, con un mínimo de repeticiones 4 y un máximo de 10, la medición del consumo de diesel se lo realizó mediante gravimetría y con el equipo de diagnóstico de Hino. El resultado obtenido de la investigación es un ahorro del 4,63 [\%] en el consumo de diesel.

Palabras claves: Eficiencia, Fuerza de arrastre, Coeficiente de arrastre, Consumo combustible, Gravimetría.

\section{Introducción.}

El consumo de energía en el Ecuador es de 93 millones de BEP (Barriles Equivalente de Petróleo), en donde el consumo de diesel alcanza un valor del 31 [\%] seguida de las gasolinas con 27 [\%] en tercer lugar se encuentra el consumo eléctrico con un valor de 15 [\%] y el consumo de gas licuado con un 9 [\%] entre los valores más altos de consumo. Considerando solo el consumo de diesel en el Ecuador es de 43 millones de BEP, de estos el 87 [\%] corresponde al sector del transporte terrestre, de los cuales la carga pesada constituye un 44 [\%], seguida de la carga liviana con un 20 [\%], razón por la cual se busca optimizar el consumo de diesel en el transporte de carga (camión y tracto-camión) (Delgado, 2016).

El parque automotor del Ecuador se subdivide en livianos con 2`127.547 unidades y comerciales con 317.370 unidades, de este último el sector de camiones tiene un valor de 228.553 unidades hasta el año 2018, razón por la cual se debe proponer alternativas de mejora que permitan reducir el consumo de diesel a nivel país (AEADE, 2020).

El camión experimenta un sin número de fuerzas que se oponen al movimiento entre las más conocidas se encuentra la fuerza a la rodadura delantera y posterior, fuerza a la pendiente 
superable debido a una componente del propio peso del camión adicionando el peso de la carga, la fuerza a la inercia esta es debido a que el camión parte del reposo y la fuerza aerodinámica (arrastre) que aparece cuando el camión incrementa su velocidad, desplazando una gran cantidad de aire, que fluye tanto exteriormente como interiormente las cavidades del camión, ocasionando fenómenos de turbulencia que generan inestabilidad

Un cuerpo en movimiento, inmerso en un fluido experimenta fuerzas ocasionadas por la acción del fluido. El efecto total de estas fuerzas es muy complejo. Sin embargo, para propósitos de diseño o estudio del comportamiento de un cuerpo en un fluido, son dos las fuerzas resultantes de mayor importancia es el arrastre y la sustentación. Sin que importe, si es el cuerpo el qué se mueve en el fluido o el fluido el qué se mueve alrededor del cuerpo.

El arrastre es la fuerza sobre un cuerpo ocasionado por el fluido que opone resistencia en la dirección del movimiento del cuerpo. Las aplicaciones más comunes que requieren el estudio del arrastre se dan en el campo del transporte. La resistencia al viento es el término que se emplea con frecuencia para describir los efectos del arrastre sobre las aeronaves, automóviles, camiones y trenes. La fuerza de arrastre debe contrarestarse por medio de una fuerza de propulsión en dirección opuesta, con el fin de mantener o incrementar la velocidad del vehículo.

La sustentación es una fuerza ocasionada por el fluido en dirección perpendicular a la dirección del movimiento del cuerpo. Su aplicación más importante está en el diseño y análisis de alas de los aeroplanos. La geometría de un aeroplano es tal que se produce una fuerza de sustentación cuando el aire pasa sobre y bajo él. La magnitud de la sustentación debe ser al menos igual al peso de la aeronave para que vuele.

Los carenados aerodinámicos tienen un impacto notable en la resistencia aerodinámica. El carenado frontal solo puede reducir alrededor del $17 \%$ de la resistencia. Es posible una mayor reducción del arrastre de hasta el 26\% utilizando varias combinaciones de carenados aerodinámicos en diferentes partes de la carrocería del camión. (Chowdhury, y otros, 2013)

En Europa se ha establecido un proceso para medir el consumo de combustible de un camión mediante una relación entre la densidad del diesel y las emisiones contaminantes (hidrocarburos, monóxido de carbono y dióxido de carbono), esto bajo un ciclo de prueba estandarizado para un banco dinamométrico (CEPE R101, 2007).

En Alemania se utiliza un tramo de carretera de $10[\mathrm{~km}]$ que debe cumplir varios requerimientos tanto atmosféricos como geográficas, el camión debe tener buenas condiciones mecánicas, en especial la presión de inflado y el estado del labrado de los neumáticos. El camión debe circular con una velocidad constante de $90[\mathrm{~km} / \mathrm{h}]$, por tanto el conductor debe en lo posible repetir el mismo estilo de conducción en todas las pruebas, el 
método de medición se lo realiza por gravimetría al inicio y final de cada prueba (DIN 70 030-2, 1986).

En Estados Unidos de América, se utiliza dos camiones de similares características, uno de ellos llevaría las modificaciones y el otro es de control, ambos recorren una distancia de 64 $[\mathrm{km}]$, con una velocidad constante de $90[\mathrm{~km} / \mathrm{h}]$, el método de medición es por gravimetría a al inicio y final de cada prueba, esto se logra con la implementación de un tanque de combustible adicional portable con conexiones de acople rápido, que servirá para una fácil traslado hacia la balanza donde se determinará su masa, una vez llegado a los puntos de control y registro de datos, al final se establece una relación entre los dos camiones y se obtiene el porcentaje de ahorro del diesel (SAE J1321, 2012).

Estudios realizados en Chile indican que utilizando varios dispositivos aerodinámicos (carenado superior, frontal y lateral, generador de vórtices y cubre tanque) sobre un tractocamión y bajo los requerimientos de la norma SAE J1321, se obtiene una reducción del 15.4 $\%$ del consumo de diesel de un tracto-camión (Villalobos, Arancibia, Retamal, Olivio, \& Vasquez, 2011).

Una alternativa a la implementación de dispositivos aerodinámicos en el camión, es trabajar directamente en el diseño de cabina, como lo muestra un estudio realizado por Chile en donde se considera 3 camiones de similares características, uno de control y dos de prueba, una de ellos dispone de una cabina modificada, las pruebas se rigen por los requerimientos de la norma SAE J1321, obteniendo un valor de 3,96 [\%] en el ahorro del consumo de diesel (Villalobos, Salazar, Gavilan, \& Rojas, 2012).

Un deflector de techo de la cabina proporciona un ahorro de combustible del 2 al 4 [\%], al revestir el flujo de aire sobre el espacio entre el tractor y el remolque. Es una placa plana o curva ajustable que se adhiere al techo del tractor y se inclina hacia el remolque. (Ministerio Energía, 2009)

\section{Metodología.}

El camión que se utiliza es un Hino 500 del año 2014, que dispone de un conector de diagnóstico que permite la comunicación con el software ECO 100 que registra varios parámetros (velocidad del vehículo, temperatura del motor, presión atmosférica, etc.) de cada una de las pruebas. El dispositivo aerodinámico utilizado es un carenado superior (rompevientos) elaborado por la Carrocera Suntaxi, que se ajusta a las dimensiones de la cabina y la zona de carga del camión, a diferencia del modelo único que ofrece la marca Hino, como se observa en la figura 1. 


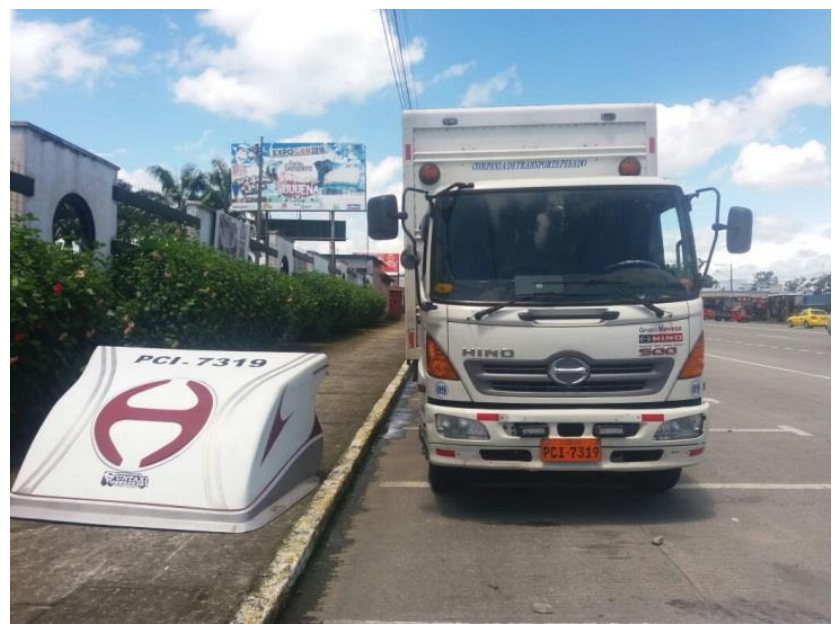

Figura 1. Camión y deflector de aire

Fuente: Elaboración propia

Se recurre a una norma Alemana para la determinación del consumo de diesel, de manera que se debe cumplir ciertos requerimientos que se establecen en las pruebas, ajustándose a las condiciones locales (atmosféricas, geográficas, calzada, estado del camión, tipo de diesel, etc), a continuación se indica varios de estos requisitos de la norma (DIN 70 030-2, 1986):

- Distancia de prueba de $10[\mathrm{~km}]$

- Pendiente de la carretera menor a 1,5 [\%]

- La diferencia de altitud entre el punto de partida y llegada sea de \pm 150 [m] (+ ascendente, - descendente)

- La velocidad del viento menor a $3[\mathrm{~m} / \mathrm{s}]$

- Presión atmosférica de 991 a 1017 [hPa]

- Temperatura del aire de 10 a $30\left[{ }^{\circ} \mathrm{C}\right]$

- La velocidad de prueba sea 3/4 de la velocidad máxima ( $\approx 90[\mathrm{~km} / \mathrm{h}])$

- Método gravimétrico para medir el consumo de combustible

- Porcentaje de repetibilidad menor al 5 [\%]

- Carretera sin presencia de lluvia

- Repetir la prueba un mínimo de 4 veces y un máximo de 10

La ruta de prueba que cumple con los requerimientos establecidos por la norma, es el tramo de carretera en la vía Santo Domingo - La Concordia, que cuenta con una superficie de hormigón y se encuentra en buen estado, además la distancia entre los radares de control de velocidad es la óptima para evitar inconvenientes con las infracciones de tránsito, la presencia de tráfico es minina a ciertas horas, es una vía de cuatro carriles lo que facilita la circulación vehicular y existe el espacio suficiente para detenerse a realizar los registros de datos de las pruebas, los equipos utilizados para el registro de los datos de las condiciones atmosféricas de la ruta, trazado de la ruta y la medición del consumo de diesel son: un anemómetro, 
altímetro, balanza digital, tanque de combustible portátil, equipo de diagnóstico Hino y un odómetro, esto se indica en la figura 2.
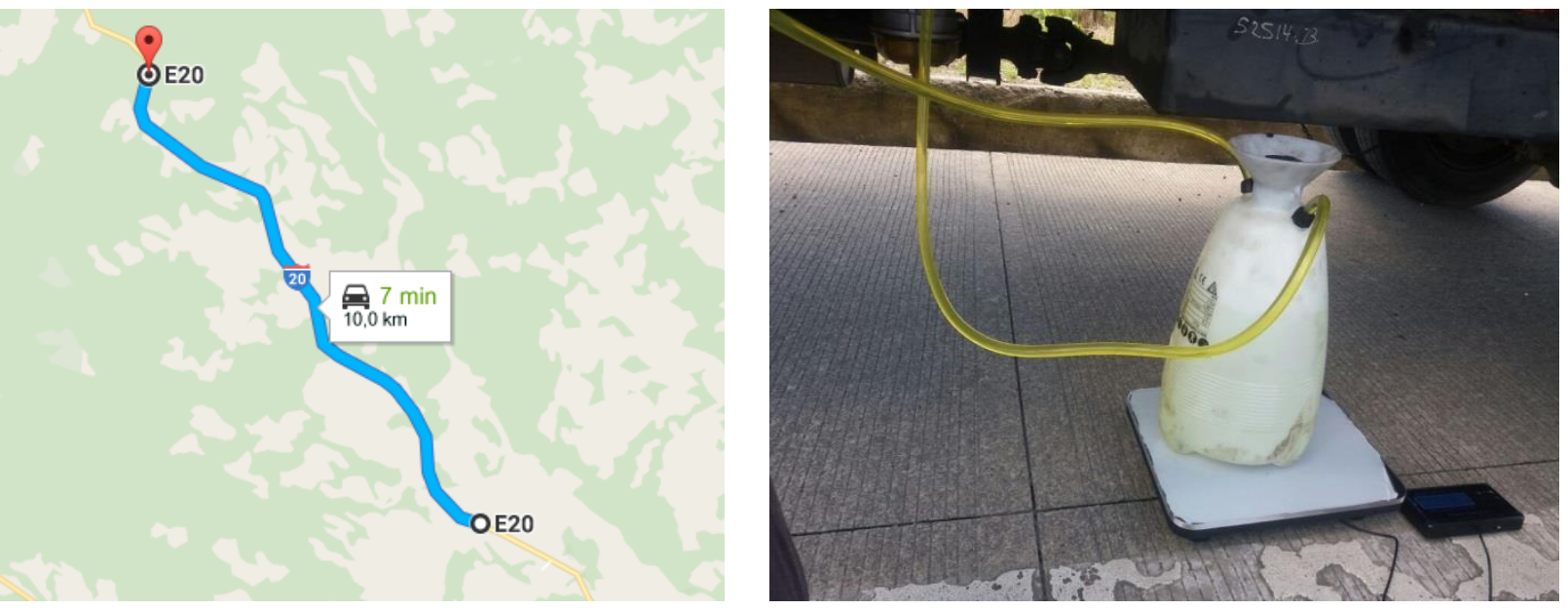

Figura 2. Ruta de prueba y la medición por gravimetría

Fuente: Elaboración propia

Al desarrollar el protocolo de prueba, se tiene que medir la masa del combustible al inicio de la prueba (punto A), el camión alcanza los $90[\mathrm{~km} / \mathrm{h}]$ en el punto $\mathrm{B}$, mantiene la velocidad hasta llegar al punto $\mathrm{C}$, luego disminuye su velocidad hasta detenerse en el punto $\mathrm{D}$, donde nuevamente se mide la masa del combustible como se indica la figura 3 , la diferencia e medidas nos da el consumo de combustible en kilogramos como sugiere el método gravimétrico.

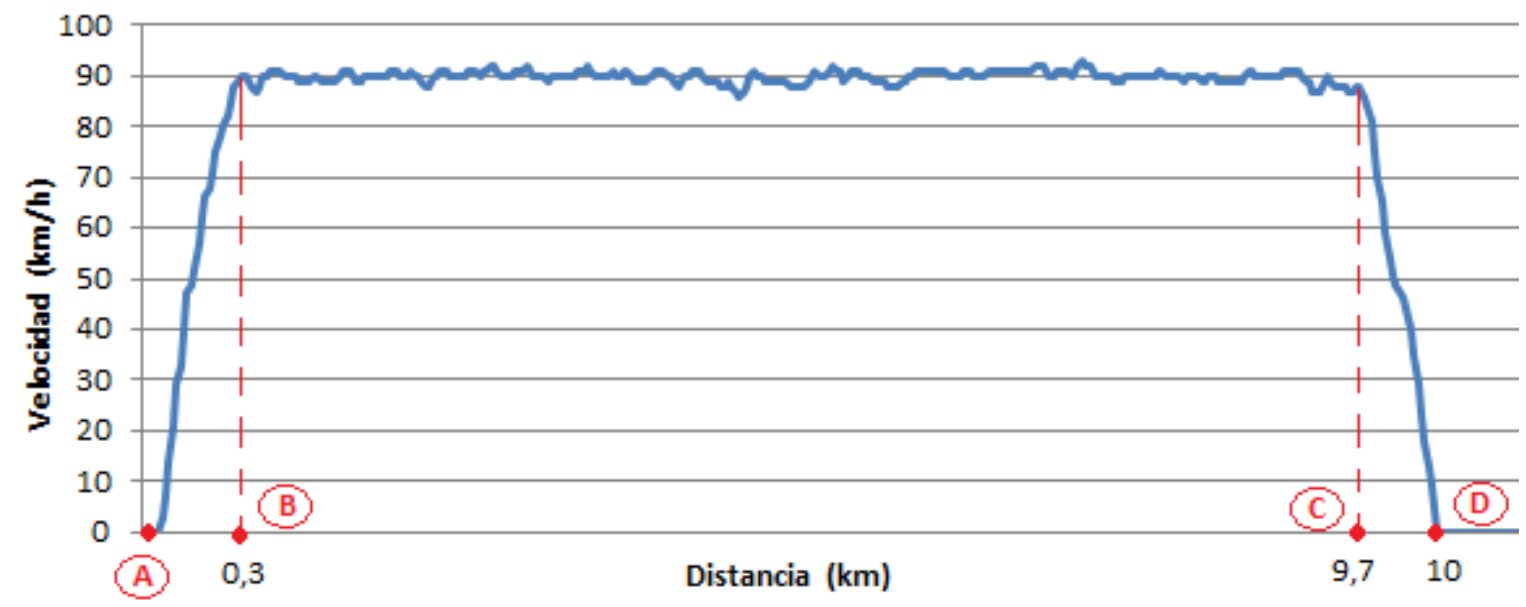

Figura 3. Protocolo de prueba

Fuente: Elaboración propia 


\section{Resultados}

Las condiciones mecánicas del camión son las más idóneas, el estado de los neumáticos es óptimo, la presión de los neumáticos se encuentra según lo recomienda el fabricante (manual de usurario) tanto para las ruedas delanteras como posteriores. Se procede a desconectar las conexiones del tanque de combustible y sustituirlo por el tanque desmontable, verificando en todo momento el normal funcionamiento del motor, se realiza una prueba por toda la ruta con todos los equipos de manera de comprobar el funcionamiento de las mismas y que el conductor ajuste su estilo de conducción a una velocidad constante, para dar inicio a las pruebas.

El estado de la vía en el día que se realizó las pruebas fue óptimo sin la presencia de lluvia, sin ningún tipo de restricción tanto de obras públicas como de tránsito, se inicia registrando los valores correspondientes a las condiciones atmosféricas y geográficas que se indican en la tabla 1 .

Tabla 1. Condiciones atmosféricas

\begin{tabular}{ll}
\hline \multicolumn{1}{c}{ Descripción } & \multicolumn{1}{c}{ Valor } \\
\hline Velocidad del viento & $1,5[\mathrm{~m} / \mathrm{s}]$ \\
Temperatura del aire & $29,3\left[{ }^{\circ} \mathrm{C}\right]$ \\
Presión atmosférica & $950,1[\mathrm{hPa}]$ \\
Presencia de lluvia & $\mathrm{NO}$ \\
Diferencia de altitud & $-106[\mathrm{~m}]$ \\
\hline
\end{tabular}

Fuente: Elaboración propia

Colocar la balanza en una superficie plana para acerar el equipo, esto se lo debe llevar a cabo en cada una las mediciones que se realicen. A de más se requiere que el aire acondicionado este apagado así como cualquier accesorio innecesario dentro del camión, Todas las pruebas deben realizarse bajo las mismas condiciones, en la parte geográfica se considera la diferencia de altitud, si es positiva se realizan todas las pruebas en sentido de ascenso y si es negativa se harán en el sentido de descenso.

Las mediciones del consumo de combustible del camión con el deflector de aire, por el método gravimétrico se indican en la tabla 2 
Tabla 2. Consumo de combustible con deflector de aire

\begin{tabular}{lllll}
\hline $\mathbf{N}^{\circ}$ Prueba & Hora inicio & $\mathbf{m}_{\mathbf{o}}(\mathbf{k g})$ & $\mathbf{m}_{\mathbf{f}}(\mathbf{k g})$ & $\Delta_{\mathbf{m}}(\mathbf{k g})$ \\
\hline 1 & $10: 38: 00$ & 8,15 & 6,80 & 1,35 \\
2 & $11: 11: 00$ & 8,65 & 7,35 & 1,30 \\
3 & $11: 38: 00$ & 8,35 & 7,10 & 1,25 \\
4 & $12: 05: 00$ & 8,80 & 7,55 & 1,25 \\
\hline
\end{tabular}

Fuente: Elaboración propia

Las mediciones del consumo de combustible del camión sin el deflector de aire, por el método gravimétrico se indican en la tabla 3

Tabla 3. Consumo de combustible sin deflector de aire

\begin{tabular}{crrrr}
\hline $\mathbf{N}^{\circ}$ Prueba & Hora inicio & $\mathbf{m}_{\mathbf{o}}(\mathbf{k g})$ & $\mathbf{m}_{\mathbf{f}}(\mathbf{k g})$ & $\Delta_{\mathbf{m}}(\mathbf{k g})$ \\
\hline 1 & $13: 23: 00$ & 9,25 & 7,85 & 1,40 \\
2 & $13: 51: 00$ & 8,20 & 6,85 & 1,35 \\
3 & $14: 18: 00$ & 8,50 & 7,15 & 1,35 \\
4 & $14: 46: 00$ & 7,80 & 6,50 & 1,30 \\
\hline
\end{tabular}

Fuente: Elaboración propia

El porcentaje de repetibilidad no debe exceder el 5 [\%], para ello se utiliza la expresión (DIN 70 030-1, 1990):

$$
R=k * \frac{z}{\sqrt{n}} * \frac{100}{\bar{C}}
$$

En donde:

$R:$ Repetibilidad [\%]

$k$ : Factor [adimensional]

$z$ : Factor $[\mathrm{kg}]$

$n$ : Número de mediciones

$\bar{C}:$ Media aritmética de $\mathrm{n}$ mediciones $[\mathrm{kg}]$

Este porcentaje tiene un valor de 4,838 [\%] para el camión sin deflector de aire y un valor de 5,949 [\%] en el camión con deflector de aire, razón por la cual se recomienda realizar una prueba adicional. 
La densidad del diesel premium tiene un valor de $826,2\left[\mathrm{~kg} / \mathrm{m}^{3}\right]$ obtenida a una temperatura de 15 [ ${ }^{\circ} \mathrm{C}$ ], mediante un ensayo bajo la norma ASTM D 287 (Valencia, 2013), al utilizar la siguiente expresión se obtiene el volumen de diesel consumido:

En donde:

$$
v=\frac{m}{\delta}
$$

$v:$ Volumen $\left[\mathrm{m}^{3}\right]$

$m$ : Masa [kg]

$\delta:$ Densidad $\left[\mathrm{kg} / \mathrm{m}^{3}\right]$

Se realiza una transformación de unidades, para obtener el consumo de diesel en litros, de manera que el valor promedio de todas pruebas es de 1,634 [litros] sin deflector de aire y un valor de 1,559 [litros] con deflector de aire.

La norma Alemana propone la siguiente expresión para determinar el consumo de combustible en camiones y buses.

$$
C=\frac{1,1 * V * 100}{s}
$$

En donde:

$C$ : Consumo de combustible [litro/100 km]

$V$ : Volumen de combustible consumido [litro]

$s$ : Distancia recorrida $[\mathrm{km}]$

El camión sin deflector de aire consume 17,978 [litros/100km] de diesel, esto hace referencia a que se requiere 17,978 litros de diesel para recorrer una distancia de $100 \mathrm{~km}$; el valor al utilizar el deflector de aire es de 17,146 [litros/100km], el ahorro del consumo de diesel es $\operatorname{del} 4,63[\%]$.

El equipo de diagnóstico Hino, registra varios datos (hora, rpm, velocidad del vehículo, voltaje sensor del acelerador 1 y 2, presión atmosférica, porcentaje de posición del pedal de acelerador 1 y 2, cantidad de inyección y temperatura del refrigerante del motor), cada segundo transcurrido. En promedio cada prueba tiene una duración de 6 [min] en los cuales, el total de la cantidad inyectada de diesel en cada una de las pruebas.

El registro de datos con el equipo de diagnóstico Hino del camión con el deflector de aire se indica en la tabla 4. 
Tabla 4. Registro de datos con deflector de aire

\begin{tabular}{|c|c|c|c|c|c|c|c|c|c|c|}
\hline $\begin{array}{c}\mathbf{N}^{\circ} \\
\text { Prueb } \\
\mathbf{a}\end{array}$ & rpm & $\begin{array}{c}\text { Velocida } \\
\text { d } \\
\text { vehículo } \\
(\mathrm{km} / \mathrm{h})\end{array}$ & $\begin{array}{l}\text { Voltaje } \\
\text { sensor } \\
\text { acelerado } \\
\text { r No. 1 } \\
\text { (voltio) }\end{array}$ & $\begin{array}{l}\text { Voltaje } \\
\text { sensor } \\
\text { acelerado } \\
\text { r No. } 2 \\
\text { (voltio) }\end{array}$ & $\begin{array}{c}\text { Presión } \\
\text { atmosféric } \\
\text { a (kPa) }\end{array}$ & $\begin{array}{c}\text { Porcentaj } \\
\text { e posición } \\
\text { pedal } \\
\text { acelerado } \\
\text { r \#1 }(\%)\end{array}$ & $\begin{array}{c}\text { Porcentaj } \\
\text { e posición } \\
\text { pedal } \\
\text { acelerado } \\
\text { r \#2 }(\%)\end{array}$ & $\begin{array}{c}\text { Cantida } \\
\mathbf{d} \\
\text { inyecció } \\
\mathbf{n} \\
\text { (mg/stk) }\end{array}$ & $\begin{array}{c}\text { Temperatur } \\
\text { a } \\
\text { refrigerante } \\
\text { motor }\left({ }^{\circ} \mathbf{C}\right)\end{array}$ & $\begin{array}{c}\text { Cantida } \\
\text { d } \\
\text { inyecció } \\
\text { n (gal) }\end{array}$ \\
\hline 1 & 1853 & 78,073 & 2,175 & 2,053 & 96,034 & 46,968 & 42,241 & 29,858 & 83,141 & 0,347 \\
\hline 2 & 1906 & 80,941 & 2,275 & 2,145 & 96,008 & 49,855 & 45,010 & 30,517 & 83,070 & 0,353 \\
\hline 3 & 1968 & 84,196 & 2,329 & 2,195 & 95,802 & 52,243 & 46,916 & 31,333 & 83,014 & 0,353 \\
\hline 4 & 1965 & 84,123 & 2,319 & 2,186 & 95,961 & 51,585 & 46,315 & 30,718 & 82,966 & 0,346 \\
\hline $\begin{array}{l}\text { Prome } \\
\text { dio }\end{array}$ & 1923 & 81,833 & 2,274 & 2,145 & 95,951 & 50,163 & 45,121 & 30,607 & 83,048 & 0,350 \\
\hline
\end{tabular}

Fuente: Elaboración propia

El registro de datos con el equipo de diagnóstico Hino del camión sin el deflector de aire se indica en la tabla 5.

Tabla 5. Registro de datos sin deflector de aire

\begin{tabular}{|c|c|c|c|c|c|c|c|c|c|c|}
\hline $\begin{array}{c}\mathbf{N}^{\circ} \\
\text { Prueb } \\
\mathbf{a}\end{array}$ & rpm & $\begin{array}{l}\text { Velocida } \\
\text { d } \\
\text { vehículo } \\
(\mathbf{k m} / \mathbf{h})\end{array}$ & $\begin{array}{c}\text { Voltaje } \\
\text { sensor } \\
\text { acelerado } \\
\text { r No. 1 } \\
\text { (voltio) }\end{array}$ & $\begin{array}{c}\text { Voltaje } \\
\text { sensor } \\
\text { acelerado } \\
\text { r No. } 2 \\
\text { (voltio) }\end{array}$ & $\begin{array}{c}\text { Presión } \\
\text { atmosféric } \\
\text { a (kPa) }\end{array}$ & $\begin{array}{l}\text { Porcentaj } \\
\text { e posición } \\
\text { pedal } \\
\text { acelerado } \\
\text { r \#1 (\%) }\end{array}$ & $\begin{array}{l}\text { Porcentaj } \\
\text { e posición } \\
\text { pedal } \\
\text { acelerado } \\
\text { r \#2 }(\%)\end{array}$ & $\begin{array}{c}\text { Cantida } \\
\text { d } \\
\text { inyecció } \\
\mathbf{n} \\
\text { (mg/stk) }\end{array}$ & $\begin{array}{c}\text { Temperatur } \\
\text { a } \\
\text { refrigerante } \\
\text { motor }\left({ }^{\circ} \mathbf{C}\right)\end{array}$ & $\begin{array}{c}\text { Cantida } \\
\text { d } \\
\text { inyecció } \\
\text { n (gal) }\end{array}$ \\
\hline 1 & 1934 & 82,637 & 2,339 & 2,207 & 95,566 & 52,418 & 47,335 & 33,653 & 83,107 & 0,378 \\
\hline 2 & 1949 & 83,124 & 2,335 & 2,202 & 95,645 & 52,228 & 47,091 & 32,827 & 83,132 & 0,378 \\
\hline 3 & 1942 & 82,717 & 2,314 & 2,181 & 95,621 & 51,750 & 46,527 & 32,093 & 83,157 & 0,368 \\
\hline 4 & 2002 & 86,355 & 2,372 & 2,235 & 95,585 & 53,511 & 48,369 & 33,235 & 83,103 & 0,372 \\
\hline $\begin{array}{l}\text { Prome } \\
\text { dio }\end{array}$ & 1957 & 83,708 & 2,340 & 2,206 & 95,604 & 52,477 & 47,330 & 32,952 & 83,125 & 0,374 \\
\hline
\end{tabular}

Fuente: Elaboración propia

Al comparar los datos obtenidos, se observa una diferencia en la velocidad que se registrada en camión por parte del equipo de diagnóstico en cada una de las pruebas, esto es debido a que se depende directamente de las destrezas del conductor y que las pruebas se las realizó en una carretera de uso público, a pesar que en el velocímetro del panel de instrumentos en todo momento marco los $90[\mathrm{~km} / \mathrm{h}]$ considerando que es un instrumento analógico. Los valores correspondientes a la cantidad de diesel inyectado durante cada una de las pruebas se indican el figura 4. 


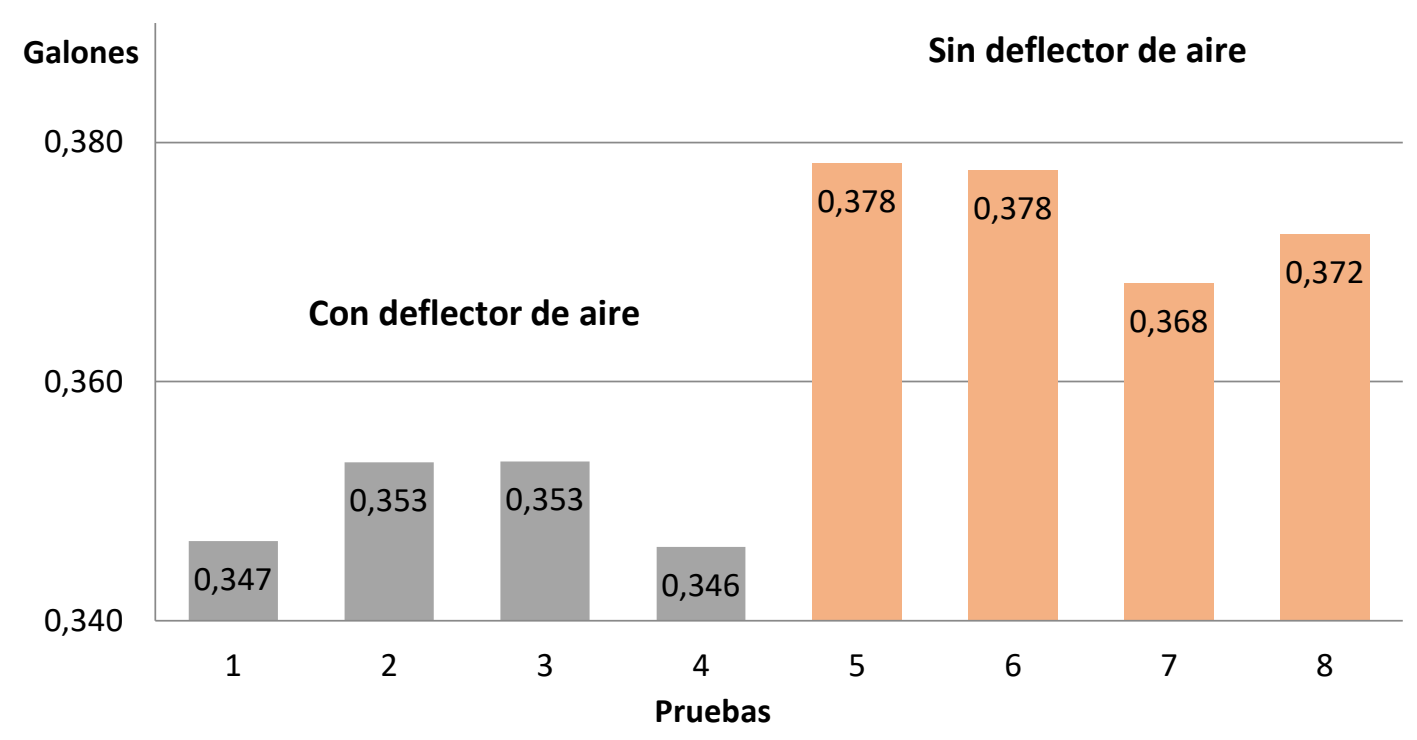

Figura 4. Datos del equipo de diagnóstico Hino

Fuente: Elaboración propia

En promedio sin la utilización el deflector de aire, el combustible consumido es de 0,374 [Galones] y con el deflector de aire tiene un valor de 0,350 [Galones], al realizarse los cálculos se obtiene un ahorro del consumo de diesel del 4,7 [\%] similar al obtenido aplicando la norma Alemana.

\section{Conclusiones.}

- Acorde a la experiencia del conductor del camión, se notó una ligera inestabilidad al momento de realizar las pruebas sin del deflector de aire, ocasionado por el aire que golpea directamente en la parte superior de la cabina, generando turbulencias a lo largo del camión.

- La ruta de prueba y los procesos de toma de datos, se ajustan a las estipulaciones expuestas por la norma Alemana, de tal manera que los resultados obtenidos son similares al equipo de diagnóstico Hino, con un 4,63 [\%] en el ahorro del diesel.

- Al año un camión ha recorrido cien mil kilómetros en promedio, de manera que la cantidad de diesel ahorrada es aproximadamente 232,77 [Galones], considerando el número de camiones que circulan por el territorio ecuatoriano representa un ahorro importante en el consumo de diesel.

- Aislar totalmente los factores externos (curvas en la carretera, ligeros desniveles, presencia de vehículos, cansancio del conductor, etc) es realmente complicado, por esta razón se tiene que realizar varias pruebas a fin descartar valores fuera de rango. 
- La gran variedad de dispositivos aerodinámico que se pueden implementar en un camión o tracto-camión, influyen directamente en el porcentaje de ahorro del consumo de diesel, razón por la cual se debe continuar con los estudios de estos dispositivos.

\section{Referencias bibliográficas.}

AEADE. (2020). Sector automotor en cifras. Quito: AEADE.

CEPE R101. (2007). Medición de la emisión de dioxido de carbono y consumo de carburante. 69.

Chowdhury, H., Moria, H., Ali, A., Khan, I., Alam, F., \& Watkins, S. (2013). Estudio aerodinámico del arrastre en un semi-trailer. Australia: ELSEVIER.

Delgado, D. (2016). Balance Energético Nacional. Quito: Ministerio Coordinador de Sectores Estratégicos.

DIN 70 030-1. (1990). Método de medición del consumo de combsutible: Automóviles. 3.

DIN 70 030-2. (1986). Método de medición del consumo de combustible: camiones y buses. 1 .

Ministerio Energía. (2009). Guía aerodinámica para tractores y remolques de servicio pesado. USA: ecoTRANSPORT.

SAE J1321. (2012). Procedimiento de ensayo de consumo de combustible: camiones y buses.

Valencia, L. (2013). Caracterización de Mezclas Diesel - Biodiesel. Quito: UCE.

Villalobos, J., Arancibia, N., Retamal, S., Olivio, P., \& Vasquez, J. (2011). Impacto de la Aerdinámica para el Ahorro de Combustible. Chile: ACHEE.

Villalobos, J., Salazar, C., Gavilan, C., \& Rojas, C. (2012). Impacto del diseño de cabinas en el consumo de combustible. Chile: ACHEE.

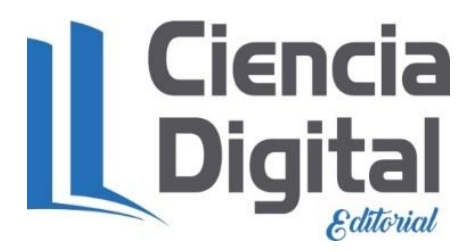




\section{PARA CITAR EL ARTÍCULO INDEXADO.}

Pachacama Gualotuña, D. P., Pachacama Gualotuña, D. A., Vinlasaca Viera, L. P., \& Castillo Medina, A. G. (2020). Eficiencia en el consumo de diésel de un camión con la implementación de un dispositivo aerodinámico. Ciencia Digital, 4(2), 6-18. https://doi.org/10.33262/cienciadigital.v4i2.1193

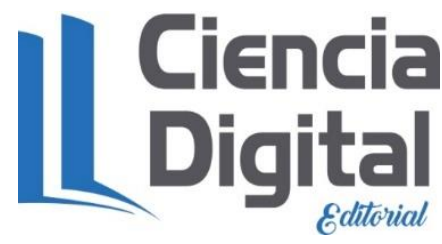

El artículo que se publica es de exclusiva responsabilidad de los autores y no necesariamente reflejan el pensamiento de la Revista Ciencia Digital.

El artículo queda en propiedad de la revista y, por tanto, su publicación parcial y/o total en otro medio tiene que ser autorizado por el director de la Revista Ciencia Digital.
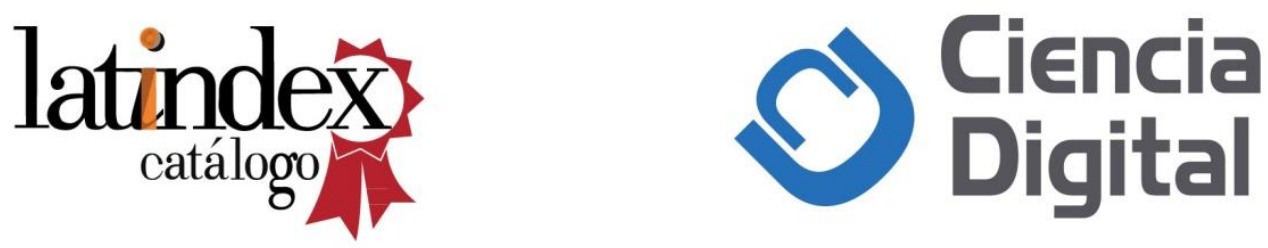